\title{
Modelling pulsed releases for sterile insect techniques: fitness costs of sterile and transgenic males and the effects on mosquito dynamics
}

\author{
Steven M. White ${ }^{1 *} \dagger$, Pejman Rohani ${ }^{2,3,4}$ and Steven M. Sait ${ }^{1}$ \\ ${ }^{1}$ Institute of Integrative and Comparative Biology, Faculty of Biological Sciences, University of Leeds, Leeds LS2 9JT, \\ UK; ${ }^{2}$ Department of Ecology and Evolutionary Biology, University of Michigan, Ann Arbor, MI 48109, USA; ${ }^{3}$ Center \\ for the Study of Complex Systems, University of Michigan, Ann Arbor, MI 48109, USA; and ${ }^{4}$ Fogarty International \\ Center, National Institutes of Health, Bethesda, MD 20892, USA
}

\section{Summary}

1. The development of transgenic technologies, coupled with sterile insect techniques (SIT), is being explored in relation to new approaches for the biological control of insect pests. Recent studies have shown that there are often fitness costs associated with transgenic insect strains, but the impact of these costs on their potential use in pest control is poorly understood.

2. In this paper, we explore the impact of an insect fitness cost on two control strategies (classical SIT and transgenic late-acting bisex lethality) using a stage-structured mathematical model, which is parameterized for the mosquito Aedes aegypti. Counter to the majority of studies, we use realistic pulsed release strategies and incorporate a fitness cost, which is manifested as a reduction in male mating competitiveness.

3. For both models we show that the level of control of a pest mosquito population is highly sensitive to the rate at which the transgenic or sterile males are released. Population control is more effective when smaller numbers of sterile/transgenic males are released more frequently than larger and less frequent releases.

4. If the wild-type mosquito population exhibits cycles of peaks and troughs in abundance, as is the case for many insect species, then high frequency releases of transgenic males not only reduce mosquito abundance, but they may dampen future pest outbreaks, whereas the use of SIT alone may have an adverse effect, causing an increase in mosquito abundance. Additionally, the timing of sterile/transgenic male release during the mosquito population cycle is critical in reducing pest outbreak levels.

5. In all cases, the reduced fitness of the sterile/transgenic males causes reductions in control, thus requiring more frequent or greater magnitude releases.

6. Synthesis and applications. The sterile insect technique is considered to be a valuable non-chemical tool for pest management. With the potential application of recent genetic developments to enhance the technique, it is becoming increasingly important to consider the wider ecological implications of this biological control strategy. Predicting the most efficient release strategies will be important in combating pest and vector insects as well as for limiting potential broader ecological effects. Although the focus of our models are based on the mosquito, A. aegypti, which can spread yellow fever, dengue fever and Chikungunya disease, our modelling approach and results can be applied more broadly to other species.

Key-words: Aedes aegypti, biological control, dengue fever, fitness, population cycles, RIDL, SIT, transgenic

*Correspondence author. E-mail: smwhit@ceh.ac.uk $\uparrow$ Present address: Centre for Ecology \& Hydrology, Maclean Building, Benson Lane, Crowmarsh Gifford, Wallingford, Oxfordshire OX10 8BB, UK.

\section{Introduction}

For centuries, humans have attempted to control insect populations. This is in part because of the significant mortality and morbidity burden associated with insect vector-borne diseases 
(Manga 2002; Gubler 2004), but also due to the huge economic impact of insect pests leading to losses in global food production (Pimentel et al. 2000; Pimentel 2007). While a variety of control approaches have been explored, they have commonly been chemically-based. However, as a result of (i) the everpresent risk of pest resistance to chemical pesticides, (ii) the increasing pressure for countries to adopt a low carbon economy in the future, and (iii) the desire for environmentally benign approaches to pest control, alternative methods, such as biological control, are increasingly sought (Dyck, Hendrichs \& Robinson 2005). One environmentally friendly alternative is the sterile insect technique (SIT) (Knipling 1955). This speciesspecific method of insect control relies on the mass rearing, sterilization and release of large numbers of male insects (Dyck, Hendrichs \& Robinson 2005), which - it is hoped mate with wild-type females, thereby reducing their reproductive output, and potentially the pest population abundance. Mixed sex sterile releases are generally not considered as it is less efficient and for some species, such as mosquitoes, it is only the females that bite and therefore could potentially aid disease spread in the short-term [see Alphey et al. (2010) for a recent review]. This approach has achieved some success in controlling several insect pest species, including screwworm (mixed sex method), Mediterranean fruit flies, and tsetse flies (Krafsur 1998; Benedict \& Robinson 2003). Overall, however, it is generally agreed that classical SIT strategies have had sporadic successes, leading to the recent development of transgenic technologies, such as genetic sexing (Robinson, Franz \& Fisher 1999), genetic marking (Peloquin et al. 2000) and genetic female-specific lethality (Seawright et al. 1978) to improve SIT efficacy (Benedict \& Robinson 2003; Wimmer 2003; Alphey et al. 2010). For example, transgenic techniques can increase the efficiency and reliability of male-only mass production (Heinrich \& Scott 2000; Thomas et al. 2000) or they may be used to induce molecular sterility instead of radiation (Alphey \& Andreasen 2002; Alphey et al. 2008), which often leads to reduced mating fitness (Helinski, Parker \& Knols 2009).

One such transgenic strategy is release of insects carrying a dominant lethal (RIDL) (Thomas et al. 2000; Fu et al. 2010). The principle of RIDL is such that if the released transgenic insects are homozygous for a dominant lethal and mate with wild insects, all the progeny are heterozygous for a dominant lethal and so die, in the case of bisex RIDL strategies. Sex specific (often female-specific (Fu et al. 2010)) RIDL strategies have also been developed, but these are not considered here. The stage at which the dominant lethal is expressed (e.g. embryo, larva, etc.) can have a significant effect on the control strategy (Phuc et al. 2007), with late-acting (i.e. death occurring after the density-dependent larval stage) having a significant advantage over its early-acting counterpart and SIT because of an additional reduction in pest abundance as a result of larval competition. Thus, depending on the stage of induced death, SIT and bisex RIDL strategies rely on similar principles, but are based on very different technology (Phuc et al. 2007; Yakob, Alphey \& Bonsall 2008).

Whilst fitness costs due to radiation have often dogged SIT control (Helinski, Parker \& Knols 2009), recent studies have shown that there are often fitness costs associated with transgenic insect strains when compared with their wild-type counterparts (Catteruccia, Godfray \& Crisanti 2003; Irvin et al. 2004; Moreira et al. 2004; Marrelli et al. 2006). These fitness costs are crucial because the transgenic insects must compete effectively with the local populations to either introgress effector genes into the wild gene pool, in the case of gene-drive systems, or to sexually compete with wild-type males, in the case of transgenic aided SIT or RIDL. These fitness costs are often construct- and strain-specific, they occur across a range of fitness components and the magnitude of the cost may vary considerably. For example, Irvin et al. (2004) examined the reproductive and developmental fitness of three transgenic lines of Aedes aegypti relative to non-transgenic mosquitoes. Their results showed that all the lines analysed had high fitness costs; survivorship was significantly reduced for all life stages; fecundity was considerably decreased and adult longevity was lower in two lines. One criticism of this result is that the fitness cost may be a consequence of inbreeding (Marrelli et al. 2006). In another example Moreira et al. (2004) examined the fitness of Anopheles stephensi mosquitoes carrying two different transgenes that inhibit the transmission of Plasmodium berghei (a rodent parasite used as a model organism for the study of human malaria). They found a significant reduction in fitness (fecundity) in one transgene strain via life-table analysis and a quick loss of the transgene allele in caged experiments, whilst the other strain showed no such fitness reduction. In contrast, Morrison et al. (2009) found little difference in the mating competitiveness of two RIDL strains of Mediterranean fruit fly, Ceratitis capitata. The impact of the fitness costs associated with transgenic insects and their potential for use in pest control is poorly understood [but see Yakob, Alphey \& Bonsall (2008)], largely because of the lack of relevant fitness studies and field data, whether this is because of constraints on the release of transgenic insects or difficulties associated with obtaining long-term ecological data in the field.

Mathematical models have been used to answer important questions about SIT since the 1950s (Knipling 1955; Berryman 1967; Barclay \& Mackauer 1980; Lewis \& van den Driessche 1993; Phuc et al. 2007; Tyson et al. 2008; Yakob, Alphey \& Bonsall 2008), and the development of appropriate mathematical models can potentially answer important ecological and pest control problems more generally. Typically, the models define a pest population in a single equation as either a discrete time difference equation [see Knipling (1955) for example] or as a continuous time differential equation [see Barclay \& Mackauer (1980) for example], to which sterile insects are released at a constant level to reduce the pest population. The critical release rate (the minimum rate of sterile releases required to eradicate the pest population) is then calculated. However, these models fail to take into account the stagestructured life-history of the insects, which can have significant effects on their dynamics, [but see Barclay (1980) and Esteva \& Mo Yang (2005) for example] as found in many other models of insect population dynamics (Wearing et al. 2004; White, Sait \& Rohani 2007), where short-period population oscillations in abundance occur from the developmental lags between 
life-history stages (Murdoch, Briggs \& Nisbet 2003). In the case of late-acting bisex RIDL, since the late-acting system kills the heterozygous offspring after the density-dependent larval stage, additional mortality occurs in the wild-type population. This has been found to have a significant control effect over classical SIT and early-acting bisex RIDL systems (Atkinson et al. 2007; Phuc et al. 2007; Yakob, Alphey \& Bonsall 2008). Therefore, the strength of density dependence and the functional form in which it occurs is likely to have a large effect on the level of population control (Atkinson et al. 2007; Yakob, Alphey \& Bonsall 2008).

In most control models, whether SIT or transgenic, it is often assumed that the release rate of sterile/transgenic males is constant, whereas in reality, the release may come in the form of a pulse or multiple pulses, since the insects are mass-reared and released at periodic times. Therefore, it is critical to understand the effects of the release frequency and abundance has on the target population, and how they interact given the complex developmental life-history.

In this paper, we explore the impact of two control strategies: SIT and late-acting bisex RIDL (referred to as RIDL hereafter), which assume fitness costs, using models. Both models are stage-structured, and are parameterized for the mosquito, A. aegypti. We apply pulsed sterile/transgenic male releases at different frequencies, which cause reductions in wild-type mosquito abundance that are highly sensitive to the rate of sterile/transgenic insect male release. However, we also demonstrate that, under some circumstances, the pulsed release of sterile/transgenic male insects can give rise to increases in wild-type abundance. We also consider the timing of release of sterile/transgenic males when the wild-type mosquito population exhibits cycles in abundance and investigate the effects of variation in fitness costs (manifested as a variation in mating competitiveness in the transgenic strain) on the effectiveness of SIT/RIDL biological control strategies.

\section{Materials and methods}

The stage-structured model we use to study the wild-type mosquito population is derived from the framework presented by Dye (1984), where $N(t)$ is the population of female wild-type mosquitoes at time $t$. We assume that mosquito population growth is stage-structured and that density dependence occurs at the larval stage (Dye 1984), with associated parameters $\alpha$ and $\beta$. As discussed by Dye (1984), this two parameter density dependence function allows for greater flexibility in the way in which density affects mortality. We also assume, for simplicity, that there is a 1:1 sex ratio in the wild-type population, and that the dynamics of the females are identical to those of the male (Phuc et al. 2007; Yakob et al. 2008). The model is described by the delayed-differential equation

$\frac{\mathrm{d} N(t)}{\mathrm{d} t}=r N(t-T) \exp \left(-\alpha(N(t-T) E)^{\beta}\right)-\mu N(t)$.

eqn 1

Here, $r$ is the daily egg production, corrected for survival from the egg to the adult stage, $T$ is the mosquito stage-structured developmental time, $E$ is the egg production rate of female adults, not corrected for density-independent survival between the egg stage and adulthood, and $\mu$ is the daily female adult death rate.
We assume that all RIDL releases carry a late-acting dominant gene, since early-acting RIDL is mathematically similar to classical SIT. For both SIT/RIDL release scenarios, wild-type female mosquitoes are assumed to mate proportionately to their relative abundance (Knipling 1955; Phuc et al. 2007), given by

$\frac{N(t)}{N(t)+c S(t)}$

where $0<c \leq 1$ is the coefficient of reduced mating competitive ability of sterile/transgenic males.

In contrast to the majority of previous studies, the SIT/RIDL control strategy takes the form of pulsed releases, where the sterile/transgenic mosquitoes are released at discrete times, $t=n T_{\text {rel }}$ where $n=$ $1,2,3, \ldots$. Here, $T_{\text {rel }}$ is the time between successive sterile/transgenic male mosquito releases. At each release time a constant number of sterile/transgenic male mosquitoes are released, $\theta N^{*}$, where $\theta$ is the release ratio with respect to the sterile-free equilibrium of wild-type mosquito populations, $N^{*}$.

These assumptions lead to the system of pulsed coupled delayed differential equations

$\frac{\mathrm{d} N(t)}{\mathrm{d} t}=r N(t-T)\left(\frac{N(t-T)}{N(t-T)+c S(t-T)}\right) F(t)-\mu N(t), \quad \forall t \quad$ eqn $2 \mathrm{a}$

$\frac{\mathrm{d} S(t)}{\mathrm{d} t}=-\mu S(t), \quad t \neq n T_{\text {rel }}, \quad n=1,2,3, \ldots$

eqn $2 b$

$S\left(t^{+}\right)=S(t)+\theta N *, \quad t=n T_{\text {rel }}, \quad n=1,2,3, \ldots$

eqn $2 \mathrm{c}$

where the moment immediately after the $n$th sterile/transgenic release is denoted as $t^{+}=n T_{\text {rel }}^{+}$and $F(t)$ is the larval densitydependent function. For SIT, larval competition is reduced by the fraction of successful matings by sterile males and wild-type females and hence

$F(t)=\exp \left(-\alpha\left(N(t-T)\left(\frac{N(t-T)}{N(t-T)+c S(t-T)}\right) E\right)^{\beta}\right)$. eqn $2 \mathrm{~d}$

For RIDL (late-acting), the successfully mated wild-type females and transgenic males produce offspring that develop to a post larval stage and hence add to the density-dependent mortality (Phuc et al. 2007). Thus, for RIDL the density dependence term is given by

$F(t)=\exp \left(-\alpha(N(t-T) E)^{\beta}\right)$.

eqn $2 \mathrm{e}$

It should be noted that in the absence of the sterile/transgenic male mosquito releases, the population model eqn 2 simplifies to that of Dye (1984).

The definitions of the parameters are given in Table 1.

\section{Results}

\section{CONTROL-FREE POPULATION DYNAMICS}

As demonstrated by Dye (1984), in the absence of biological control, the female mosquito population equilibrium is given by

$N *=\frac{((1 / \alpha) \log (r / \mu))^{1 / \beta}}{E}$, eqn 3

and it is stable if

$\mu T<\frac{2 \pi-\cos ^{-1}(1 / A)}{A^{2}-1}$, eqn 4 
Table 1. Parameter definitions for the mathematical model in eqn 2

\begin{tabular}{|c|c|c|}
\hline $\begin{array}{l}\text { Parameter/ } \\
\text { variable }\end{array}$ & Definition & Value \\
\hline$N$ & Number of female wild-type mosquitoes & \\
\hline$S$ & Number of male sterile or transgenic mosquitoes & \\
\hline$r$ & Birth rate of adults corrected for egg to adult survival* & 1.31 or 1.5 days $^{-1}$ when fixed \\
\hline$T$ & Mosquito development period* & $18 \cdot 84$ days \\
\hline$c$ & Coefficient of reduced competitive ability of sterile/transgenic males & 0.95 or 0.5 \\
\hline$\alpha$ & $\begin{array}{l}\text { Density-dependent coefficient ( } 1 / \alpha \text { is the size at which the population repro- } \\
\text { duces at its maximum rate) corrected for egg to adult survival } \dagger\end{array}$ & $0 \cdot 01$ \\
\hline$\beta$ & Density-dependent coefficient & 0.4 or 1 when fixed \\
\hline$E$ & Average egg production rate of females & 1 days $^{-1}$ \\
\hline$\mu$ & Adult death rate* & $0 \cdot 12$ days $^{-1}$ \\
\hline$\theta$ & Release ratio & 1 \\
\hline$N^{*}$ & Control-free female mosquito equilibrium & Given by eqn 3 \\
\hline$T_{\text {rel }}$ & Time between sterile/transgenic male releases & Varied \\
\hline
\end{tabular}

*Parameter determined by Dye (1984).

$†$ Parameters do not change the stability, but only the equilibrium size of the wild-type only dynamics and so are scaled to give reasonable population sizes.

where $A=1-\beta \ln (r / \mu)$. Hence, the parameters $E$ and $\alpha$ only have an effect on the equilibrium population size, but do not have any effect on the stability of the system (Fig. 1). The controlled system eqn 2 is highly intractable, and therefore we resort to numerical integration. The delay-differential equations are solved numerically using a sixth order Runge-Kutta solver (Thompson \& Shampine 2006) with impulses (Corwin, Thompson \& White 2008).

In the control-free model eqn 1 the female adult mosquitoes exhibit stable equilibrium dynamics for low birth rates $(r)$ and density dependence $(\beta)$, as found by Dye (1984). However, greater survival during development or increased environmentally favourable conditions may lead to an increased birth rate. When the birth rate becomes sufficiently large the equilibrium loses stability, leading to periodic oscillations. The amplitude of these oscillations and the mean population abundance become increasingly large as the birth rate increases. Spectral analysis reveals that the period of the population oscillations is 51-53 days, which correspond to delayed-feedback cycles (Murdoch, Briggs \& Nisbet 2003). These cycles are a feature of

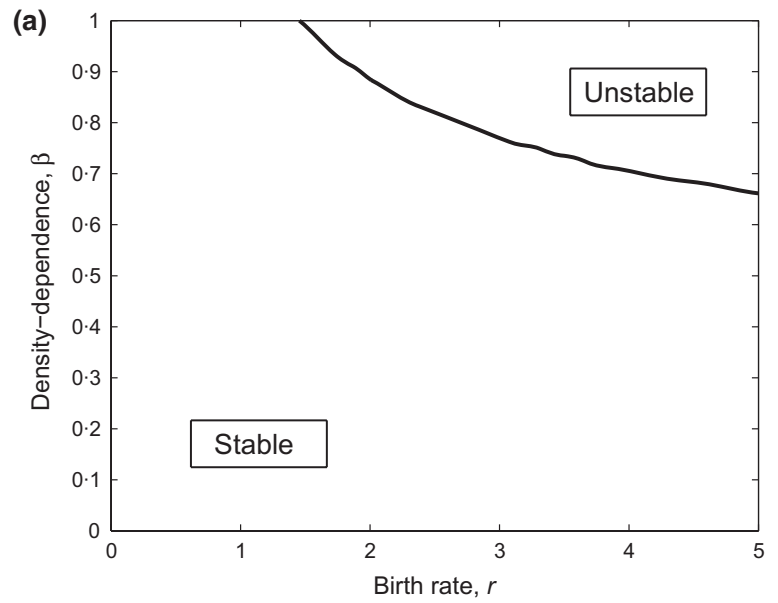

(b)

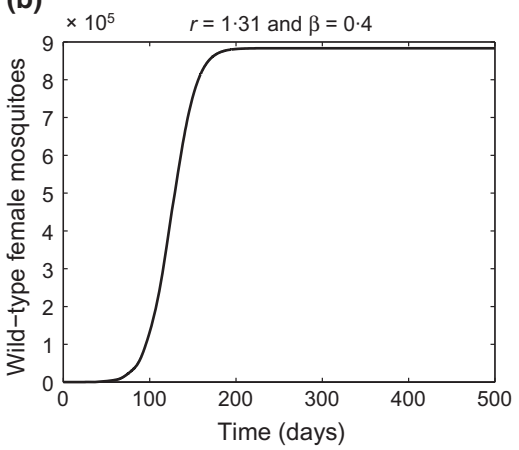

(c)

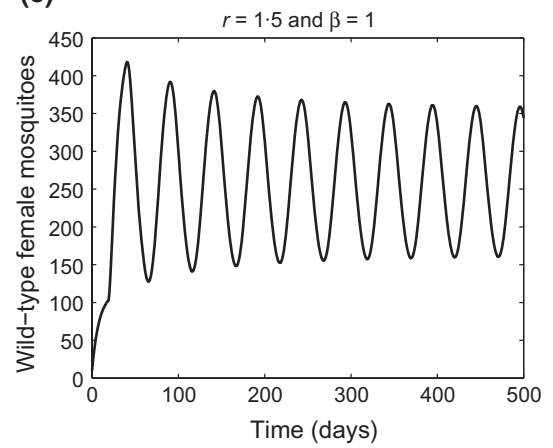

Fig. 1. The importance of the birth rate and density dependence on the control-free female mosquito dynamics. In (a) the stability criterion eqn 4 is plotted for varying values of birth rate $(r)$ and density dependence $(\beta)$. The parameter region labelled 'Stable' denotes parameter space where model eqn 1 gives rise to a stable equilibrium, whereas the parameter region labelled 'Unstable' gives rise to oscillatory dynamics. In (b) and (c) two time series are plotted for (b) $r=1.31$ and $\beta=0 \cdot 4$, which is in the stable region of parameter space; and (c) $r=1.5$ and $\beta=1$, which is just inside the unstable region. All other parameter values are given in Table 1. 
the stage-structuring (i.e. the time delay in the mathematical model) that is crucial to the development of the mosquitoes. Data collected by Sheppard et al. (1969) and Southwood et al. (1972), from which the parameter estimates are calculated and evaluated (Dye 1984), show fluctuating behaviour with an average period approximately equal to that observed by simulations of eqn 1 . Whilst other environmental factors are not included explicitly in eqn 1 , such as rainfall, the developmental structuring which occurs for mosquitoes is likely to be a major component of the observed fluctuations. Therefore, it is important that we understand how the sterile/transgenic male insects interact with these cyclical populations of wild-type mosquitoes.

\section{CONTROLLED POPULATION DYNAMICS}

\section{Equilibrium wild-type dynamics}

If the wild-type population of mosquitoes exhibit stable equilibrium dynamics (low $r$ and $\beta$ ), then the periodic releases of either sterile or transgenic males mosquitoes of any fitness will reduce the wild-type mosquito population (see Fig. 2 a,b and compare the blue solid line with the red and black solid lines). High frequency releases (low $T_{\text {rel }}$ ) will eradicate the wild-type mosquitoes for both control strategies, but the transgenic RIDL control gives greater wild-type suppression, needing less frequent releases to eradicate the population. This is due to the additional wild-type mortality caused by RIDL larval competition (Phuc et al. 2007; Yakob et al. 2008).

In contrast, low frequency releases (high $T_{\text {rel }}$ ) result in less wild-type mosquito suppression for both SIT and RIDL strategies, with RIDL out-performing SIT. However, whilst the average wild-type mosquito population is less than that for SIT at low frequency releases, the variability induced by the period release strategies are greater for the RIDL control, since density dependence is reduced for the SIT strategy.

It is clear that more frequent control releases gives greater wild-type suppression, since more sterile/transgenic insects are in the environment. However, in reality, the number of sterile/ transgenic male mosquitoes that can be released per unit of time is limited by the output of the mass-rearing factory. In practice, insects are mass-reared and released on a regular basis. An obvious question to ask is whether it would be advantageous to release half the number of sterile/transgenic insects, but twice as often? In Fig. 3 we address this question. Here, we release the same average number of sterile/transgenic male mosquitoes, but vary the frequency of releases (see Fig. 3a). We achieve this by scaling the release ratio, $\theta$, as the time between releases, $T_{\text {rel }}$, varies. For both SIT (blue lines in Fig. 3b) and RIDL (black lines in Fig. 3b), we see that more frequent and smaller releases gives greater wild-type population control. Infrequent, large releases cause large initial wildtype population suppression, but this wears off as the sterile/ transgenic insects die, allowing the wild-type mosquitoes to recover. In contrast, frequent small releases have a smaller impact, but the constant presence of the sterile/transgenic
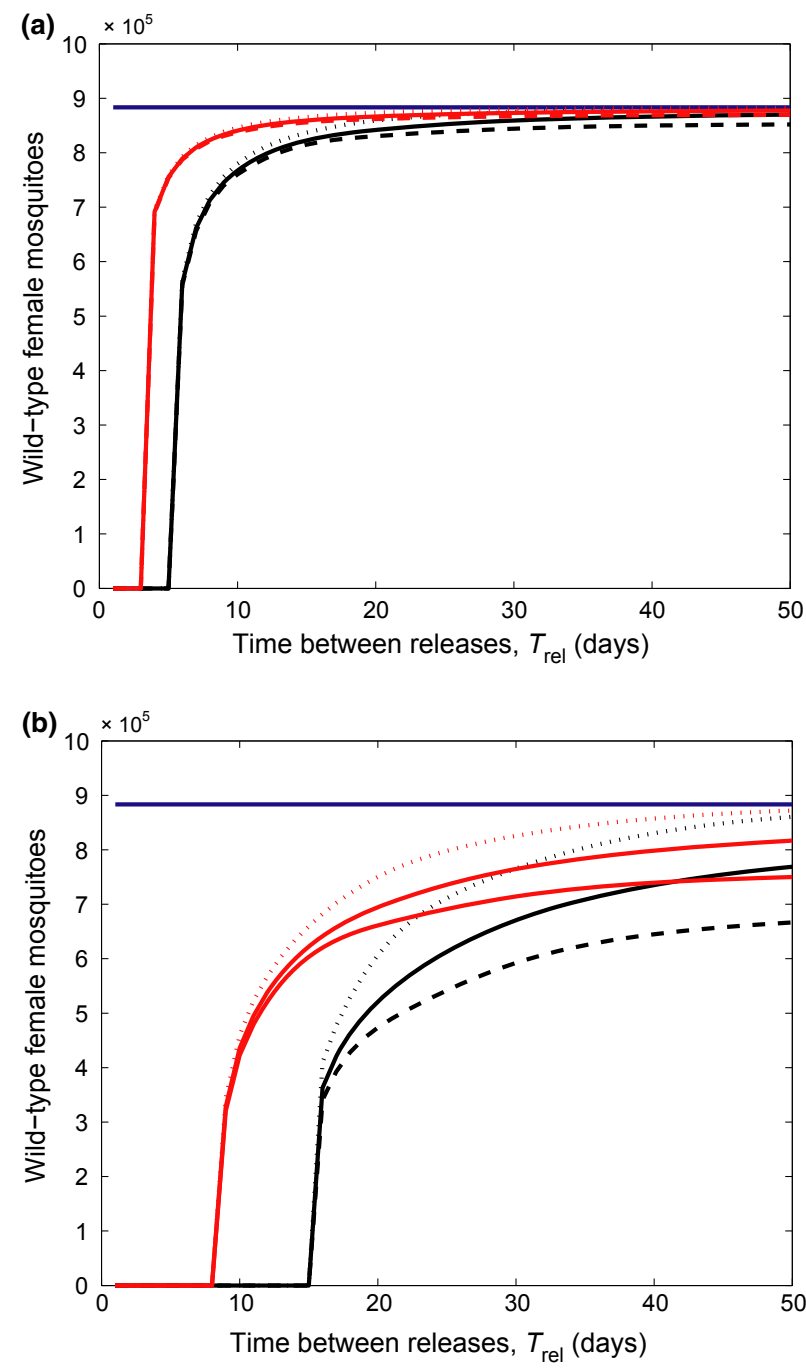

Fig. 2. The effects of varying the periodic sterile (a) and transgenic (b) male release rates for two mosquito populations with differing fitness costs on a stable wild-type mosquito population. The blue lines denotes the control-free equilibrium; the black lines denote the female wild-type mosquito population mean (solid), minimum (dashed) and maximum (dotted), where the fitness cost is low $(c=0.95)$; the red lines similarly denote the female wild-type mosquito population for a sterile/transgenic population with a high fitness cost $(c=0 \cdot 5)$. For each simulation $r=1.31$ and $\beta=0 \cdot 4$, all other parameter values are given in Table 1.

males gives greater wild-type female mosquito suppression. Moreover, this effect can be large in the sense that the frequency of releases may either lead to wild-type eradication or marginal suppression, depending on the area of parameter space, as shown here by the RIDL control strategy.

\section{Periodic wild-type dynamics}

Many species of insect exhibit cycles in population abundance (Kendall et al. 1999). Environmental drivers, such as temperature and rainfall, are often the cause of this periodicity, but there are many examples of insect populations showing shortperiod cycles that are caused by either interspecies interactions 

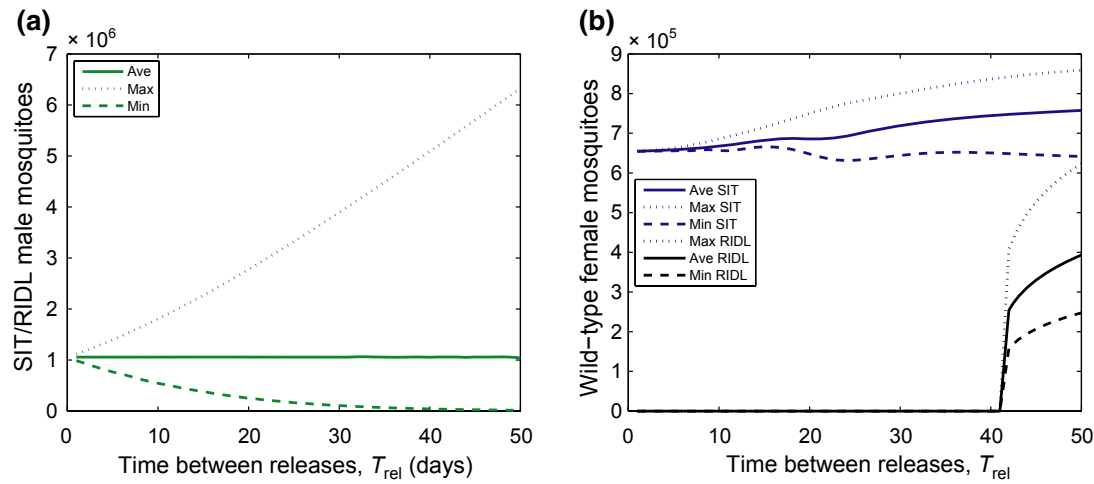

Fig. 3. The effects of varying the periodic sterile and transgenic male release rates whilst fixing the time-averaged sterile/transgenic abundance to be constant. In (a) the green lines denote the sterile or transgenic male mosquito population average (solid), minimum (dashed) and maximum (dotted), respectively, as the time between releases $\left(T_{\text {rel }}\right)$ is varied. Similarly, in (b) the corresponding wild-type female mosquito population average (solid), minimum (dashed) and maximum (dotted) as the time between releases $\left(T_{\text {rel }}\right)$ is varied for sterile insect techniques (SIT) control (blue) and release of insects carrying a dominant lethal (RIDL) control (black). In all simulations the fitness cost is assumed to be low $(c=0 \cdot 95)$. So that the average sterile/transgenic population remains constant, we scale the release ratio such that $\theta=T_{\text {rel }} / 7$. Thus, at $T_{\text {rel }}=7$ we have $\theta=1$ which gives weak wild-type suppression with an SIT control strategy and elimination with RIDL as predicted in Fig. 2. All other parameter values are given in Table 1.

(Godfray \& Hassell 1989) or by intraspecific interactions during the development (Sait, Begon \& Thompson 1994; Murdoch, Briggs \& Nisbet 2003). For A. aegypti the data collected by Sheppard et al. (1969) and Southwood et al. (1972), from which the parameter estimates are calculated (Dye 1984), show similar short-period fluctuations, although greater detail is required to determine the cause of these fluctuations.

As we have already shown, the wild-type mosquito population model exhibits such behaviours when the birth rate $(r)$ and density dependence $(\beta)$ become sufficiently large. If we assume such dynamics, and the uncontrolled wild-type mosquitoes exhibit short-period population cycles, then what effect does the time between releases have on the dynamics, and how is this affected by reduced fitness costs in the sterile/transgenic strain? In Fig. 4 we address this question.

For this region of parameter space the SIT control (see Fig. 4a) exhibits an undesirable behaviour in that releases of sterile males cause an increase in wild-type female abundance. This has previously been shown with other models (Phuc et al. 2007; Yakob et al. 2008), and occurs because the strong density dependence exhibited by the wild-type mosquitoes is reduced by the control strategy, and hence leads to an increase in abundance. Here we show that increasing the frequency of releases has a greater negative effect.

In contrast, for the RIDL strategy (see Fig. 4b) we see that any control strategy causes a decrease in the mean wild-type female mosquito population. High frequency transgenic male releases (low $T_{\text {rel }}$ ) causes wild-type mosquito eradication, as in the case of equilibrium-type dynamics. Low frequency releases result in a mean reduction in wild-type mosquitoes, but there may be peaks in adult abundance above that of the control-free scenario. This phenomenon arises from the pulse of transgenic males perturbing the wild-type mosquito dynamics, which when coupled with the stage-structure developmental delays and high density dependence, causes oscillations in mosquito abundance with increased amplitude.
Furthermore, we clearly see that any transgenic strain that suffers from an increased cost of fitness (mating competitiveness) will have reduced effectiveness on control, with greater frequency of releases required to achieve the same level of control as a similar strain with a lower fitness cost.

\section{Timing of release in cyclic populations}

Our results show that the time between releases of sterile or transgenic male mosquitoes can have a large effect on the dynamics of the wild-type mosquito population. On the other hand, if the wild-type population has cyclic dynamics, then is the best strategy to release the sterile/transgenic males at the wild-type population maximum, the minimum or some other specific time during the cycle? This may be very important for more cost-effective control strategies.

In order to determine the 'best release strategy' we must first define a suitable measure of reduced wild-type population size in controlled relative to uncontrolled scenarios. To this end we define the release effect, $R$, as

$R(t)=\frac{\int_{\tau}^{\tau+t} N_{C}(s) \mathrm{d} s}{\int_{\tau}^{\tau+t} N_{0}(s) \mathrm{d} s}$

where $N_{C}$ is the wild-type female mosquito population subject to a single sterile or transgenic release at time $\tau$, and $N_{0}$ is the uncontrolled wild-type female mosquito population. This time-dependent measure gives the relative effect of the sterile/transgenic release at different time points in the population cycle of the wild-type mosquitoes. Hence, if $R=1$ then there is no relative effect of the control strategy on the wild-type mosquito population; if $R<1$ then the release has had a (desirable) negative effect on the wild-type population; if $R>1$ then the release has had a positive effect on the wild-type population. Note that for our system, a single sterile or transgenic release results in $R \rightarrow 1$ as $t \rightarrow \infty$ since the 

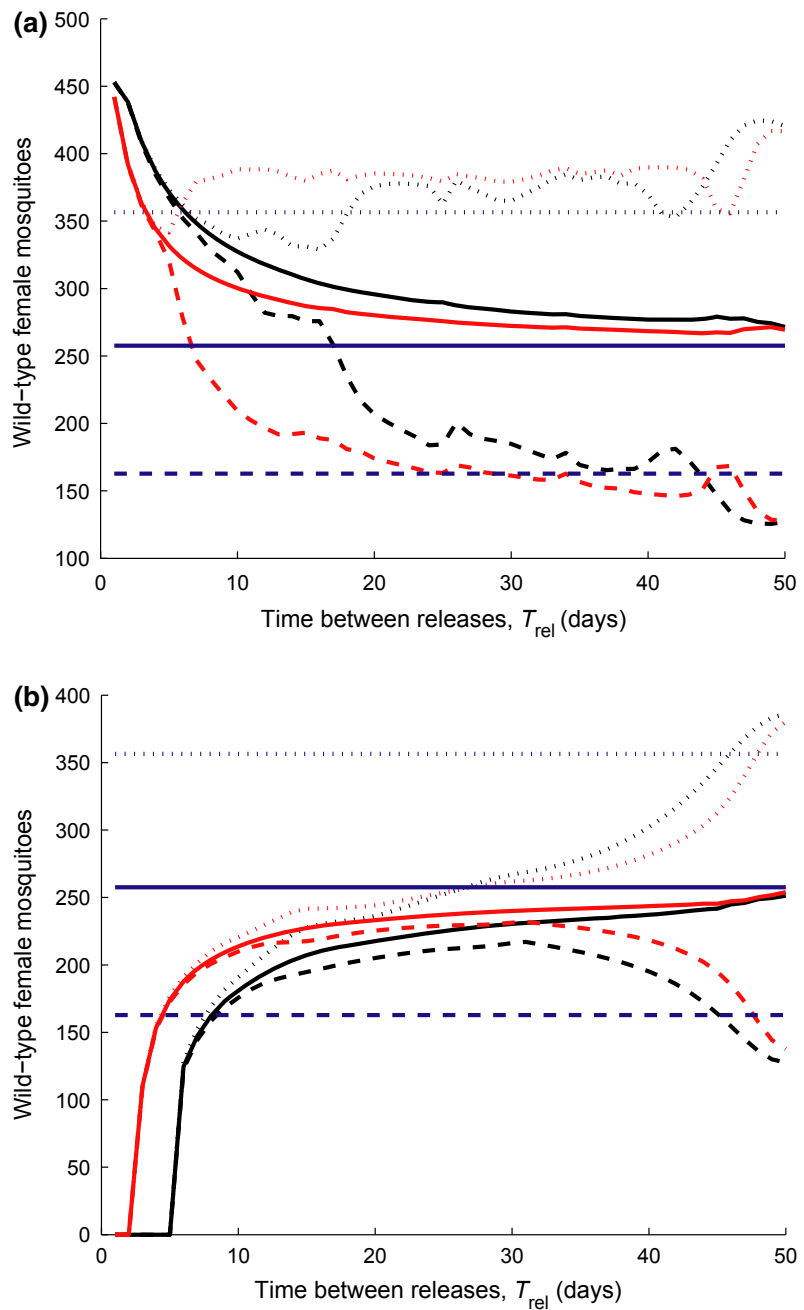

Fig. 4. The effects of varying the periodic sterile (a) and transgenic (b) male release rates for two mosquito populations with differing fitness costs on a cyclic wild-type mosquito population. All lines are as in Fig. 2 except the blue lines correspond to the mean (solid), maximum (dotted) and minimum (dashed) of the control-free periodic wild-type mosquito population. All parameters are as in Fig. 2 except $r=1.5$ and $\beta=1$.

sterile/transgenic mosquitoes eventually die-out and the dynamics return to their natural state.

In Fig. 5 we demonstrate the effect a single release of sterile or transgenic mosquitoes at different points of the wild-type population oscillation, ranging from the population minimum (pre-population peak, pure blue), to the population maximum (pure red), and back to the population minimum (post-population peak, pure green). With all release points, and for both control strategies, initially we see no immediate effects of the control on the wild-type mosquito population (Fig. 5c-d) since a reduction in population can only be seen in the next generation via the reduced numbers of progeny from mated wild-type females. It should be noted that this crucial effect would not be shown in models that do not incorporate some form of stagestructure.

As we have already discussed, the parameter region in which wild-type mosquito oscillations occur gives rise to an adverse effect using the periodic release SIT control strategy. Therefore, a single release is also likely to have an adverse effect, as shown in Fig. 5c where the timing of the release in the cycle changes the magnitude of this effect. Releases before the trough in abundance of the wild-type population cause the least adverse effect, which contrasts with a release after the wild-type peak, which causes a larger adverse effect. This is highlighted in Fig. 5(e) where these two release times are plotted, and the increase in wild-type population can be clearly seen.

For the RIDL control strategy, intuitively, one might assume that the best time to initially release transgenic males in order to have the maximum impact is at the population minimum (Benedict \& Robinson 2003), when competing wild-type males and females are low and transgenic males have a greater probability of acquiring mates. However, Fig. 5 demonstrates that the best time may be more subtle than this. Our results show that the best time to release is after the peak in adult abundance, but before the trough in wild-type mosquitoes occurs (i.e. approximately half way between the population peak and the following trough), as denoted by the large deviation in the release effect, $R$, in Fig. $5 \mathrm{~d}$. Releasing transgenic males in this way takes advantage of the relatively large numbers of fertile female mosquitoes still present in the population whilst at the same time having a larger proportional impact on a population of wild-type mosquitoes that continues to decrease towards the trough, thus suppressing the subsequent wild-type population peak. In contrast, the worst time to release the transgenic males is when the wild-type population is increasing. In Fig. 5f, we show this contrast by plotting two time series: the scenario where we observe the minimum effect (dashed lines); and the maximum effect (dotted lines). These plots highlight that, whilst keeping the number of released transgenic males constant, we are able to maximize the effect on the wild-type mosquito population by concentrating the release time to when the mosquito population is decreasing.

Releasing sterile or transgenic mosquitoes that suffer from a reduced fitness (mating competitiveness) results in smaller or larger wild-type population effects, but this does not significantly alter the best and worst time to release.

When extrapolating the release effect to multiple releases the problem becomes increasingly more difficult to interpret. First, the dynamics from multiple non-periodic sterile or transgenic mosquito releases result in complex wild-type mosquito population dynamics. Secondly, the largest deviation in the release effect occurs when the transient dynamics of any prior release have vanished and hence minimizing this function has little biological relevance, but one can simply minimize this function over the period of the short-term cycle, which we have carriedout in Fig. 6.

In Fig. 6 we see that after the first release of transgenic mosquitoes at the best release time the wild-type mosquito population has a greatly reduced subsequent outbreak level. Whilst subsequent 'best' releases may prevent further wild-type mosquito peaks, they do not cause a significant reduction in the mosquito population, since the average times between releases do not fall below the threshold for eradication, as predicted by 

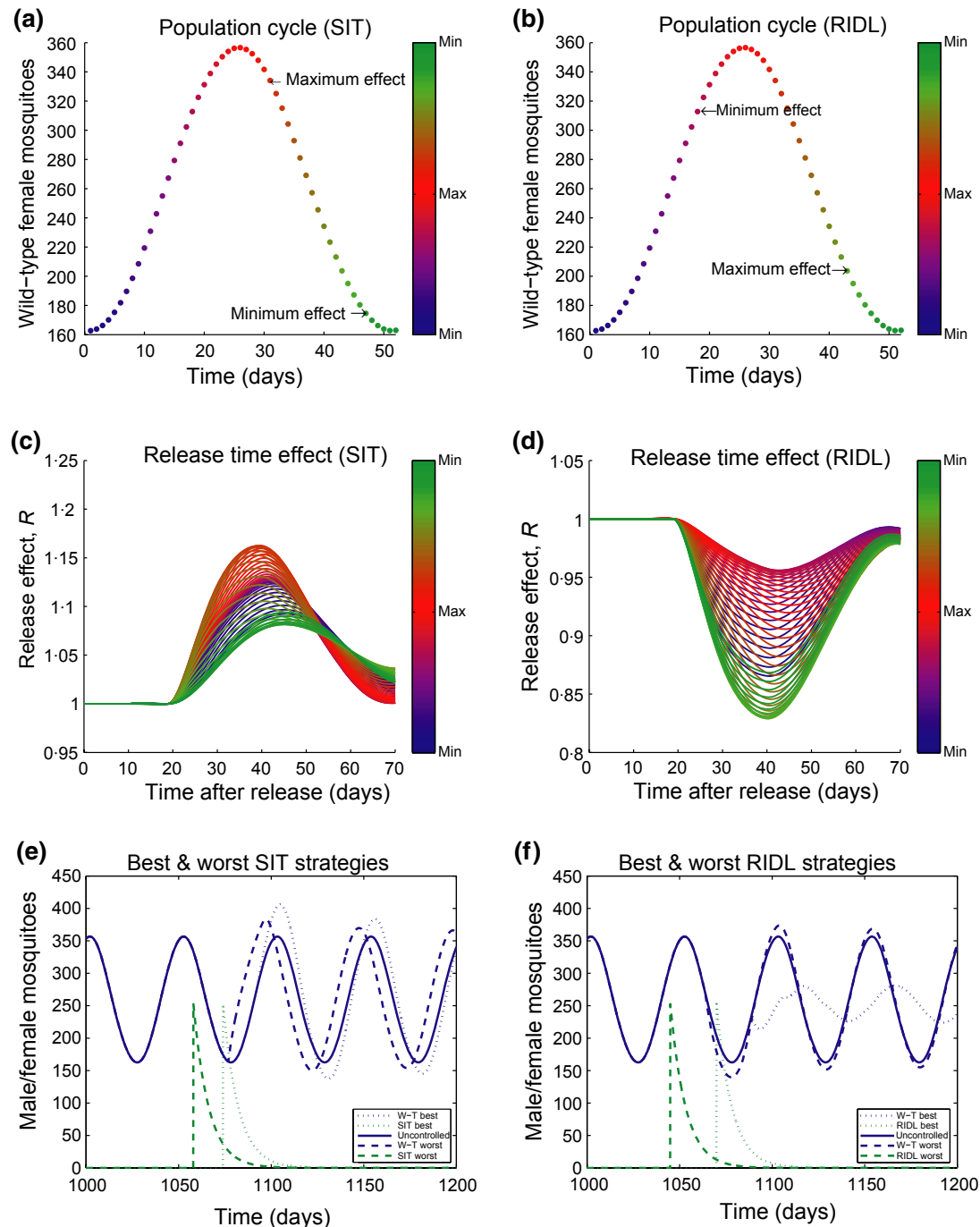

Fig. 5. The relative effect of a single sterile or transgenic release at different points throughout the wild-type mosquito population cycle. In (a) and (b) a typical pest population cycle is plotted over time, where the colours indicate different points along the cycle, ranging from the population minimum (pure blue), to the maximum (pure red) and back to the minimum (pure green). In (c) and (d) the release effect (a relative measure of deviation from the wild-type population cycle) is plotted against time for sterile insect techniques (SIT) (c) and release of insects carrying a dominant lethal (RIDL) (d) control strategies with a low fitness cost $(c=0.95)$, where the line colours denote the release effects from different points during the cycle [cf. (a) and (b)]. In (e) and (f) the time series are plotted for the minimum (dashed lines) and maximum (dotted lines) effects where for a single release of sterile or transgenic males, respectively. All parameters are given in Table 1 except $r=1.5$ and $\beta=1$.

the periodic release strategy. Therefore, these state-dependent release strategies may have to be combined with optimal control theory (Rafikov, Bevilacqua \& Wyse 2009) with a suitable control functional for efficient control.

\section{Discussion}

Our results show that the time between releases of sterile or transgenic male mosquitoes can have a large effect on the dynamics of the wild-type mosquito population, but that the control of the wild-type mosquito population is highly dependent on the rate at which the sterile/transgenic males are released, with only high release rates giving sufficient control. Counter-intuitively, we show that at lower release frequencies, stage-structured life-history effects can lead to oscillations in adult abundance that are sometimes above control-free levels, thus increasing the potential for larger than usual outbreaks in wild-type mosquito numbers. In all cases, the reduced fitness costs in the sterile or transgenic males cause significant reductions in pest control, thus requiring more frequent (and hence more costly) releases.

By modelling the more realistic periodic pulsed release strategy, we have shown that there is a benefit by adopting more frequent and smaller releases, as opposed to less frequent and larger releases. However, with more frequent releases comes increased distribution costs. Therefore, not only should one consider the control aspects in the pest management system, whether SIT or RIDL, but one should also take into account the cost/benefit relationship. Furthermore, models that assume a constant release strategy (Knipling 


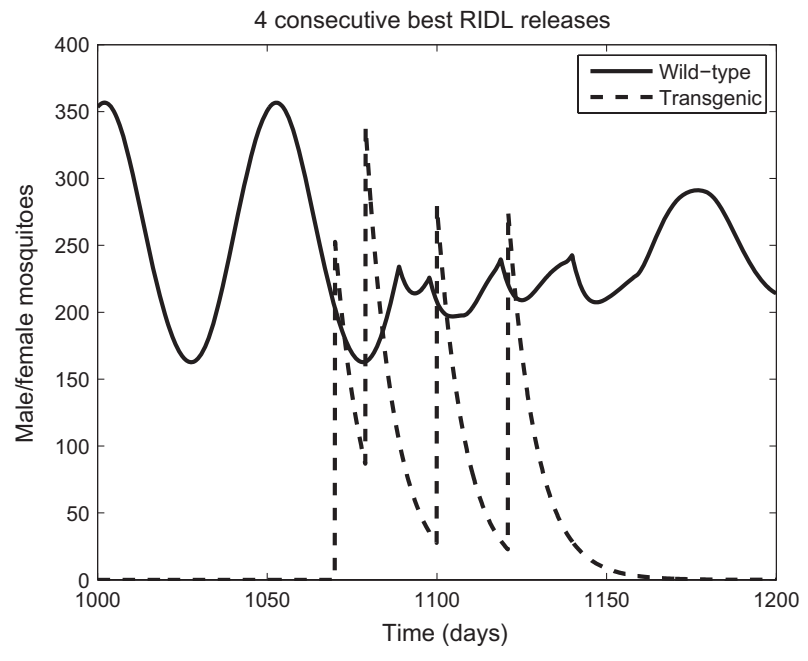

Fig. 6. The effects of four consecutive best transgenic male releases in cyclic wild-type mosquito populations. The solid line denotes the wild-type mosquito population that are subject to multiple transgenic releases at the best release time in the cycle, denoted by the dashed line. All parameter values are given in Table 1 except $c=0 \cdot 95, r=$ 1.5 and $\beta=1$.

1955; Barclay \& Mackauer 1980; Phuc et al. 2007; Yakob et al. 2008) will tend to over-estimate the true level of population control. Hence, when designing pest management programmes in general, it is important not to over-simplify the release strategy dynamics.

Our work also explores the control implications of the timing of releases, especially relevant to wild-type mosquito populations with cyclic dynamics. We demonstrate, counter to intuition, that the most effective time to release is well before the mosquito outbreak occurs (Benedict \& Robinson 2003). In other words, early intervention strategies are more effective in controlling mosquito outbreaks than late intervention. Moreover, whilst reduced fitness has an effect on the magnitude of the control success, the best time to release is nevertheless still robust. Here, we have presented a fitness cost through a reduction of mating competition, but other fitness costs may also be assumed. For example, sterile or transgenic males may carry an additional fitness cost in the form of a reduced life span (Irvin et al. 2004). Preliminary analysis shows qualitatively similar results (not presented here) as the reduced mating competition fitness cost, in that an increase in cost requires higher frequencies of sterile/transgenic males to control the mosquito population. This highlights the important point that, whilst induced fitness costs may reduce some aspects of the effectiveness of control, there may still be gains by optimizing the timing of releases. Transgenic technologies can additionally increase the efficacy of control strategies by improving mass rearing capabilities, for example (Wimmer 2003).

The parameter space required to induce the short-period cycles highlights two important points. First, the parameter estimates from Dye (1984) do not facilitate such cycles, although small changes in the upper estimate of the birth rate $(r)$ would allow such behaviour, as we have used here. However, the data from which the estimates are derived suggest such oscillations (Sheppard et al. 1969; Southwood et al. 1972), although the destabilizing mechanisms are not clearly understood. Secondly, the functional form and parameters of density dependence, which is an important factor in the cycles, relies on the accuracy and size of the data set. As Legros et al. (2009) discuss, the outcome of the functional forms and parameters may be interpreted in a significantly different way, depending on the fitting method. Therefore, we suggest that further experimental and theoretical investigation is required [but see Agnew, Haussy \& Michalakis (2000)] into the density dependence mechanisms, especially in light of the fact that our model, along with others (Phuc et al. 2007; Yakob et al. 2008), predict that SIT and early-acting - but not late-acting - RIDL control strategies may lead to an increase in wild-type mosquitoes due to over-compensatory density dependence.

The insights we have gained arise by considering a detailed model for the life-history of the wild-type species, namely the stage-structured modelling approach, which was pioneered by Gurney, Nisbet \& Lawton (1983). In contrast, whilst classical pest control models inform us of the release rates required to eradicate pest species (Knipling 1955; Murdoch, Chesson \& Chesson 1985; May \& Hassell 1988), they do not readily lend themselves to predicting release rates when dynamics are cyclical, which are generated by the discrete developmental stages (egg, larva, pupa) of the pest insects and which are frequently observed in the wild (Godfray \& Hassell 1989; Kendall et al. 1999).

For many mosquito populations, seasonal environmental effects are an important feature of their life-history, giving rise to seasonal cyclic dynamics (Mogi et al. 1998; Scott et al. 2000). For example, seasonal rainfall can increase the abundance of mosquitoes, where reproduction depends on the availability of suitable breeding sites such as water-filled containers. Moreover, many species of mosquitoes in temperate zones overwinter in a diapausal state (Mitchell 1988). Such environmental variation may give rise to seasonal bouts of infectious diseases (Altizer et al. 2006). Hence, controlling the insect vector of the infectious disease in seasonal environments must be addressed, which we aim to report on elsewhere.

Despite our emphatic results, further investigation is required to fully comprehend what the effects of multiple releases might be, since any release not only has an impact on the magnitude of any cyclic behaviour, but also on the frequency of the cycles. Thus, it is possible that some statedependent release strategies may cause unforeseen behaviours. For example, for many seasonally forced systems, the periodic forcing can react with the natural dynamics of the population, resulting in highly cyclic, or even chaotic behaviour, thus making predictions of ensuing population dynamics difficult and the possible disease outbreaks subsequently arising difficult to manage and mitigate against [see Greenman, Kamo \& Boots (2004) for example, and references therein]. Here, for cyclic wild-type mosquito populations, we considered a statedependent RIDL control where we maximized the release effect $(R)$ for different releases throughout the period of the population cycle. We showed that releasing at certain times within the cycle (before the population trough) gave 
significant improvements in population control. However, in practice, to adopt such a release strategy would require intensive monitoring, and therefore there may be a cost/benefit trade-off which should be further explored. Furthermore, the presence and nature of cycles, and the optimal release strategy relative to such cycles, may be quite sensitive to model assumptions. Nevertheless, extrapolating this result further, we predict that knowledge of environmental population drivers, such as rainfall and temperature, which also give rise to population fluctuations (Yang, Brook \& Bradshaw 2009), may be combined with specific release times to maximize control efficiency.

Whilst our model concentrates on SIT and late-acting bisex RIDL, elements may be incorporated into the closely related strategies of release of early-acting RIDL (Thomas et al. 2000; Atkinson et al. 2007 and gene drive (population replacement) (Ito et al. 2002; Rafikov, Bevilacqua \& Wyse 2009). For example, in gene drive systems the cost of the transgene may inhibit population replacement of the wild-type (Lambrechts, Koella \& Boëte 2007), which can be overcome by an increased released rate (Magori \& Gould 2006). However, these fitness shortcomings are not present in all systems (Marrelli et al. 2007).

Our models have revealed significant effects on pest mosquitoes resulting from the release of sterile or transgenic males with respect to stage-structure, release times and strategies, and fitness costs. However, since the life-history of many pest species are complex, we have adopted a simplified modelling approach in the first instance to gain a mechanistic understanding of the impact of pulsed sterile and transgenic insect releases. Several key assumptions of SIT models remain to be further examined, such as the assumption that males and females are at a constant ratio of 1:1. By definition, the mass release of sterile or transgenic males during SIT approaches disrupts this ratio, which may have an impact on the extent of mating competitiveness that sterile/transgenic males experience in the field. Incorporating greater life-history detail, such as dispersal (Otero, Schweigmann \& Solari 2008), for both wild-type and sterile/transgenic strains and their associated specific fitness costs, may prove to give greater understanding of control strategies. For example, the spatial model of Yakob et al. (2008) shows that migration of sterile males beyond the control zone may have unanticipated effects on non-target pests in the surrounding areas. Moreover, increased species and disease specific information will be required before implementing a control strategy. Microcosm and field work will help in determining specific parameters, interactions and behaviour, and combining these with relevant models will aid in the control design.

\section{Acknowledgements}

This work was carried out as a result of an International Atomic Energy Agency Co-ordinated Research Programme on 'Molecular technologies to improve the effectiveness of the sterile insect technique' (UK/12529) and we would like to thank Dr Gerald Franz and Dr Alan Robinson accordingly. This work was also partly supported by NERC grant NE/B503276/1 awarded to S.M.S. and P.R.

\section{References}

Agnew, P., Haussy, C. \& Michalakis, Y. (2000) Effects of density and larval competition on selected life history traits of Culex pipiens quinquefasciatus (Diptera: Culicidae). Journal of Medical Entomology, 37, 732-735.

Alphey, L. \& Andreasen, M. (2002) Dominant lethality and insect population control. Molecular and Biochemical Parasitology, 121, 173-178.

Alphey, L., Nimmo, D., O'Connell, S. \& Alphey, N. (2008) Insect population suppression using engineered insects. Transgenesis and the Management of Vector-Borne Disease (ed. S. Aksoy), pp. 93-103. Landes Bioscience, Austin, Texas.

Alphey, L., Benedict, M.Q., Bellini, R., Clark, G.G., Dame, D., Service, M. \& Dobson, S. (2010) Sterile-insect methods for control of mosquito-borne diseases: an analysis. Vector-Borne and Zoonotic Diseases, 10, 295-311.

Altizer, S., Dobson, A.P., Hosseini, P.R., Hudson, P.J., Pascual, M. \& Rohani, P. (2006) Seasonality and the dynamics of infectious diseases. Ecology Letters, 9, 467-484.

Atkinson, M.P., Su, Z., Alphey, N., Alphey, L.S., Coleman, P.G. \& Wein, L.M. (2007) Analyzing the control of mosquito-borne diseases by a dominant lethal genetic system. Proceedings of the National Academy of Sciences of the United States of America, 104, 9540-9545.

Barclay, H.J. (1980) Models for the sterile insect release method with the concurrent release of pesticides. Ecological Modelling, 11, 167-177.

Barclay, H. \& Mackauer, M. (1980) The sterile insect release method for pest control: a density dependent model. Environmental Entomology, 9, 810-817.

Benedict, M.Q. \& Robinson, A.S. (2003) The first releases of transgenic mosquitoes: an argument for the sterile insect technique. Trends in Parasitology, 19, 349-356.

Berryman, A.A. (1967) Mathematical description of the sterile male principle. Canadian Entomologist, 99, 858-865.

Catteruccia, F., Godfray, H.C.J. \& Crisanti, A. (2003) Impact of genetic manipulation on the fitness of Anopheles stephensi mosquitoes. Science, 299, 12251227.

Corwin, S.P., Thompson, S. \& White, S.M. (2008) Solving ODEs and DDEs with impulses. Journal of Numerical Analysis, Industrial and Applied Mathematics, 3, 139-149.

Dyck, V.A., Hendrichs, J. \& Robinson, A.S. (eds.) (2005) Sterile Insect Technique - Principles and Practice in Area-Wide Integrated Pest Management. Springer, The Netherlands.

Dye, C. (1984) Models for the population dynamics of the yellow fever mosquito, Aedes aegypti. Journal of Animal Ecology, 53, 247-268.

Esteva, L. \& Mo Yang, H. (2005) Mathematical model to assess the control of Aedes aegypti mosquitoes by the sterile insect technique. Mathematical Biosciences, 198, 132-147.

Fu, G., Lees, R.S., Aw, D.N.D., Jin, L., Gray, P., Berendonk, T.U., WhiteCooper, H., Scaife, S., Phuc, H.K., Marinotti, O., Jasinskiene, N., James, A.A. \& Alphey, L. (2010) Female-specific flightless phenotype for mosquito control. Proceedings of the National Academy of Sciences of the United States of America, 107, 4550-4554.

Godfray, H.C.J. \& Hassell, M.P. (1989) Discrete and continuous insect populations in tropical environments. Journal of Animal Ecology, 58, 153-174.

Greenman, J., Kamo, M. \& Boots, M. (2004) External forcing of ecological and epidemiological systems: a resonance approach. Physica D: Nonlinear Phenomena, 190, 136-151.

Gubler, D.J. (2004) The changing epidemiology of yellow fever and dengue, 1900 to 2003: full circle? Comparative Immunology, Microbiology \& Infectious Diseases, 27, 319-330.

Gurney, W.S.G., Nisbet, R.M. \& Lawton, J.H. (1983) The systematic formulation of tractable single-species population models incorporating age structure. Journal of Animal Ecology, 52, 479-495.

Heinrich, J.C. \& Scott, M.J. (2000) A repressible female-specific lethal genetic system for making transgenic insect strains suitable for a sterile-release program. Proceedings of the National Academy of Sciences of the United States of America, 97, 8229-8232.

Helinski, M.E.H., Parker, A.G. \& Knols, B.G.J. (2010) Radiation biology of mosquitoes. Malaria Journal, 8(Suppl. 2), S6.

Irvin, N., Hoddle, M.S., O’Brochta, D.A., Carey, B. \& Atkinson, P.W. (2004) Assessing fitness costs for transgenic Aedes aegypti expressing the GFP marker and transposase genes. Proceedings of the National Academy of Sciences of the United States of America, 101, 891-896.

Ito, J., Ghosh, A., Moreira, L.A., Wimmer, E.A. \& Jacobs-Lorena, M. (2002) Transgenic anopheline mosquitoes impaired in transmission of a malaria parasite. Nature, 417, 452-455.

Kendall, B.E., Briggs, C.J., Murdoch, W.W., Turchin, P., Ellner, S.P., McCauley, E., Nisbet, R.M. \& Wood, S.N. (1999) Why do populations cycle? A syn- 
thesis of statistical and mechanistic modeling approaches. Ecology, 80, 1789-1805.

Knipling, E.F. (1955) Possibilities of insect control or eradication through the use of sexually sterile males. Journal of Economic Entomology, 48, 459-462.

Krafsur, E.S. (1998) Sterile insect technique for suppressing and eradicating insect populations: 55 years and counting. Journal of Agricultural Entomology, 15, 303-317.

Lambrechts, L., Koella, J.C. \& Boëte, C. (2007) Can transgenic mosquitoes afford the fitness cost? Trends in Parasitology, 24, 4-7.

Legros, M., Lloyd, A., Huang, Y. \& Gould, F. (2009) Density-dependent intraspecific competition in the larval stage of Aedes aegypti (Diptera: Culicidae): revisiting the current paradigm. Journal of Medical Entomology, 46, 409-419.

Lewis, M.A. \& van den Driessche, P. (1993) Waves of extinction from sterile insect release. Mathematical Biosciences, 116, 221-247.

Magori, K. \& Gould, F. (2006) Genetically engineered underdominance for manipulation of pest populations: a deterministic model. Genetics, 172, 2613-2620.

Manga, L. (2002) Vector-control synergies, between 'roll back malaria' and the global programme to eliminate lymphatic filariasis, in the African region. Annals of Tropical Medicine and Parasitology, 96, S129-S132.

Marrelli, M.T., Moreira, C.K., Kelly, D., Alphey, L. \& Jacobs-Lorena, M. (2006) Mosquito transgenesis: what is the fitness cost? Trends in Parasitology, 22, 197-202.

Marrelli, M.T., Li, C., Rasgon, J.L. \& Jacobs-Lorena, M. (2007) Transgenic malaria-resistant mosquitoes have a fitness advantage when feeding on plasmodium-infected blood. Proceedings of the National Academy of Sciences of the United States of America, 104, 5580-5583.

May, R.M. \& Hassell, M.P. (1988) Population dynamics and biological control. Philosophical Transactions of the Royal Society B: Biological Sciences, 318, 129-169.

Mitchell, C.J. (1988) Occurrence, biology, and physiology of diapause in overwintering mosquitoes. The Arboviruses: Epidemiology and Ecology (ed. T.P. Monath), Vol. 1, pp. 191-217. CRC Press, Inc., Boca Raton, Florida, USA.

Mogi, M., Khamboonruang, C., Choochote, W. \& Suwanpanit, P. (1998) Ovitrap surveys of dengue vector mosquitoes in Chiang Mai, northern: Thailand seasonal shifts in relative abundance of Aedes albopictus and Ae. aegypti. Medical and Veterinary Entomology, 2, 319-324.

Moreira, L.A., Wang, J., Collins, F.H. \& Jacobs-Lorena, M. (2004) Fitness of anopheline mosquitoes expressing transgenes that inhibit plasmodium development. Genetics, 166, 1337-1341.

Morrison, N.I., Segura, D.F., Stainton, K.C., Fu, G., Donnelly, C.A. \& Alphey, L.S. (2009) Sexual competitiveness of a transgenic sexing strain of the Mediterranean fruit fly, Ceratitis capitata. Entomologia Experimentalis et Applicata, 133, 146-153.

Murdoch, W.W., Chesson, J. \& Chesson, P.L. (1985) Biological control in theory and practice. The American Naturalist, 125, 344-366.

Murdoch, W.W., Briggs, C.J. \& Nisbet, R.M. (2003) Consumer-Resource Dynamics. Princeton University Press, Princeton, NJ, USA.

Otero, M., Schweigmann, N. \& Solari, H.G. (2008) A stochastic spatial dynamical model for Aedes aegypti. Bulletin of Mathematical Biology, 70, 1297-1325.

Peloquin, J.J., Thibault, S.T., Staten, R. \& Miller, T.A. (2000) Germ-line transformation of pink bollworm (Lepidoptera: Gelechiidae) mediated by the piggyBac transposable element. Insect Molecular Biology, 9, 323-333.

Phuc, H.K., Andreasen, M.H., Burton, R.S., Vass, C., Epton, M.J., Pape, G., Fu, G., Condon, K.C., Scaife, S., Donnelly, C.A., Coleman, P.G., White-
Cooper, H. \& Alphey, L. (2007) Late-acting dominant lethal genetic systems and mosquito control. BMC Biology, 5, 11 .

Pimentel, D. (2007) Area-wide pest management: environmental, economic and food issues. Area-Wide Control of Insect Pests (eds. M.J.B. Vreysen, A.S Robinson \& J. Hendrichs), pp. 35-47. IAEA, Springer, The Netherlands.

Pimentel, D., Lach, L., Zuniga, R. \& Morrison, D. (2000) Environmental and economic costs of nonindigenous species in the United States. BioScience, 50, 53-65.

Rafikov, M., Bevilacqua, L. \& Wyse, A. (2009) Optimal control strategy of malaria vector using genetically modified mosquitoes. Journal of Theoretical Biology, 258, 418-425.

Robinson, A.S., Franz, G. \& Fisher, K. (1999) Genetic sexing strains in the medfly, Ceratitis capitata: development, mass rearing and field application. Trends in Entomology, 2, 81-104.

Sait, S.M., Begon, M. \& Thompson, D.J. (1994) Long-term population dynamics of the Indian meal moth Plodia interpunctella and its granulosis virus. Journal of Animal Ecology, 63, 861-870.

Scott, T.W., Morrison, A.C., Lorenz, L.H., Clark, G.G., Strickman, D., Kittayapong, P., Zhou, H. \& Edman, J.D. (2000) Longitudinal studies of Aedes aegypti (Diptera: Culicidae) in Thailand and Puerto Rico: population dynamics. Journal of Medical Entomology, 37, 77-88.

Seawright, J.A., Kaiser, P.E., Dame, D.A. \& Lofgren, C.S. (1978) Genetic method for the preferential elimination of females of Anopheles albimanus. Science, 200, 1303-1304

Sheppard, P.M., Macdonald, W.W., Tonn, R.J. \& Grab, B. (1969) The dynamics of an adult population of Aedes aegypti in relation to dengue haemorrhagic fever in Bangkok. Journal of Animal Ecology, 38, 661-702.

Southwood, T.R.E., Murdie, G., Yasuno, M., Tonn, R.J. \& Reader, P.M. (1972) Studies on the life budget of Aedes aegypti in Wat Samphaya, Bangkok, Thailand. Bulletin of the World Health Organization, 46, 221226.

Thomas, D.D., Donnelly, C.A., Wood, R.J. \& Alphey, L.S. (2000) Insect population control using a dominant, repressible lethal, genetic system. Science, 287, 2474-2476.

Thompson, S. \& Shampine, L.F. (2006) A friendly Fortran DDE solver Applied Numerical Mathematics, 56, 503-516.

Tyson, R., Newton, K.D., Thistlewood, H. \& Judd, G. (2008) Mating rates between sterile and wild codling moths (Cydia pomonella) in springtime: a simulation study. Journal of Theoretical Biology, 258, 319-330.

Wearing, H.J., Sait, S.M., Cameron, T.C. \& Rohani, P. (2004) Stage-structured competition and the cyclic dynamics of host-parasitoid populations. Journal of Animal Ecology, 73, 706-722.

White, S.M., Sait, S.M. \& Rohani, P. (2007) Population dynamic consequences of parasitised-larval competition in stage-structured host-parasitoid systems. Oikos, 116, 1171-1185.

Wimmer, E.A. (2003) Applications of insect transgenesis. Nature Reviews Genetics, 4, 225-232.

Yakob, L., Alphey, L. \& Bonsall, M.B. (2008) Aedes aegypti control: the concomitant role of competition, space and transgenic technologies. Journal of Applied Ecology, 45, 1258-1265.

Yang, G., Brook, B.W. \& Bradshaw, C.J.A. (2009) Predicting the timing and magnitude of tropical mosquito population peaks for maximizing control efficiency. PLoS Neglected Tropical Diseases, 3, e385.

Received 18 June 2010; accepted 18 August 2010

Handling Editor: Jennifer Firn 